\title{
La atención de la salud en Argentina: Al gran pueblo argentino... ¿Salud? - Primera entrega
}

Carlos García ${ }^{\ddagger}$

\begin{abstract}
Resumen
En esta primera, de una serie de entregas relacionadas, el autor describe mediante la utilización de viñetas clínicas utilizadas a modo de ejemplo, los diferentes factores que hacen de la administración de salud argentina una "tragedia" (en este caso falencias relacionadas a la falta de regionalización y atención por niveles). Y describe, a su vez, cómo la estructuración y utilización correcta de los recursos disponibles podrían llevar a consolidar un sistema nacional de salud eficiente y costo-efectivo.
\end{abstract}

\section{Abstract}

In this first release of a series of related articles, the author describes (using clinical vignettes), the different factors that turns the Argentinian health administration into a "tragedy" (in this case, shortcomings related to the lack of regionalization and health care levels). He describes, in turn, how the structure and proper use of available resources could lead to consolidate an efficient and cost effective national health system.

García C. La atención de la salud en Argentina: Al gran pueblo argentino... ¿Salud? - Primera entrega. Evid Act Pract Ambul. 2016;19(1):2-4.Ene-Mar

En la Argentina es abrumadora la cantidad de factores que hacen que la cobertura de salud sea muy deficiente. $Y$ peor aún, es el hecho de que utilizando en forma racional y planificada los recursos disponibles, la atención podría mejorar en forma significativa.

La atención de la salud en Argentina, como muchos otros países, tiene características tan particulares que hacen imposible hablar de un sistema de salud. En la Argentina conviven, compiten y se superponen, múltiples sistemas de financiación de la salud que generan una dilapidación continua de recursos. De ser estos bien empleados, mejorarían muchos indicadores y también la satisfacción de muchos de sus ciudadanos, aunque, claro está, no todos. La implementación de un verdadero sistema nacional de salud, más equitativo y centrado en la población, mejoraría la salud y la satisfacción gran parte de la población, a la vez que, inevitablemente, atentaría contra intereses muy importantes concentrados en pocas personas.

Una proporción significativa del gasto en salud se realiza en el ámbito de las obras sociales provinciales y las obras sociales sindicales cuyas prestaciones son de calidad muy irregular, la accesibilidad es limitada, no existe la figura del médico de cabecera, ni una historia clínica informatizada y el acceso a los medicamentos también es irregular. Obras sociales de unos pocos miles de afiliados montan recursos para cientos de miles que luego no son utilizados. Cada vez que se quiere hacer propaganda política, se abre un hospital de "alta complejidad y moderna tecnología" que luego no se usa porque los profesionales trabajan en la medicina privada o no tienen la mínima capacitación para operar dignamente un equipo de última generación.

Como anécdota, muy ilustrativa, cabe mencionar un hospital regional de una zona de población de bajos recursos que adquirió dos equipos, para realizar cirugía robótica. La cirugía robótica y su utilidad y las ventajas sobre la cirugía convencional aún no están plenamente demostradas, la formación profesional para el manejo de un robot requiere muchas horas y práctica, el costo de un robot, su mantenimiento y su amortización son altísimos y la utilización de esos recursos en áreas donde la mortalidad infantil está aún a niveles muy altos es inadmisible. Se requiere mucha formación, mucha investigación, recorrer mucho camino y asignar ingentes recursos antes de que la cirugía robótica sea un recurso sine qua non en los servicios de salud. Sin embargo municipios o provincias argentinas como Malvinas Argentinas o Misiones han comprado robots que muy pocos sabrán o estarán en condiciones de utilizar o lo que es peor, se utilizarán en condiciones en las que su ventaja es harto dudosa a expensas de los recursos de poblaciones que se beneficiarían de prácticas mucho más razonables y costo efectivas ${ }^{1-3}$.

En los aproximadamente doscientos países que tiene el mundo, debe haber alrededor de doscientos "sistemas" de salud diferentes. Sin embargo, si restringimos la utilización de la calificación de "sistema" a la definición de "Conjunto ordenado de normas y procedimientos que regulan el funcionamiento de un grupo o colectividad. ", muy pocos países quedarán comprendidos en este calificativo.

El mejor de los sistemas de salud, tendrá defectos, muchos aspectos para corregir y mucho camino por recorrer, de la misma forma que muchos de los peores sistemas pueden plantear algunas soluciones útiles y costo-efectivas dignas de imitar.

La medicina general (o medicina familiar como se denomina en Estados Unidos) es a menudo descrita como "la gema en la corona" del Sistema Nacional de Salud Inglés (NHS por National Health System) y contribuye a que este sistema clasifique muy alto en comparaciones internacionales en tres aspectos. A nivel de pacientes individuales provee cuidados para individuos con las condiciones de salud más comunes con un creciente énfasis en pacientes con múltiples condiciones coexistentes y un compromiso de continuidad y cuidado integral de los individuos. A nivel poblacional la práctica general sirve a comunidades locales definidas, de manera que se puede centrar en la prevención y en la educación así como el tratamiento de las enfermedades. $Y$ a nivel del sistema de salud, la medicina general brinda cuidados eficientes manejando el riesgo clínico a través de sus servicios centrales previniendo la sobre-medicalización, facilitando el acceso apropiado a los servicios especializados y de investigación y abogando por los pacientes toda vez que esto es necesario ${ }^{4,5}$.

En una serie de notas que comienzo en esta publicación iré describiendo mediante la utilización de viñetas clínicas a modo de ejemplo, los diferentes factores que hacen de nuestra administración de salud una tragedia y que de ser correctamente estructurados podrían hacernos estar orgullosos de tener un sistema nacional de salud eficiente y costo efectivo. En estas notas describiré la viñeta, comentaré cómo se desarrolla en la Argentina y cómo se desarrollaría en el Reino Unido utilizando a este país como comparación simplemente porque es uno de los sistemas de salud más eficientes y equitativos del mundo. 
Marcelo tiene 55 años y vive en una ciudad de la Patagonia, se comunica telefónicamente conmigo, que vivo en Buenos Aires y me cuenta que desde hace 20 días tiene dolor en el pecho. Lo interrogo como suelo interrogar todos los dolores, con la herramienta altamente sofisticada del acrónimo ALICIA (A: en qué circunstancias aparece; L: dónde le duele; I: adónde se irradia; C: las características; I: la intensidad y A: qué cosas lo agravan, cuáles lo atenúan y qué otros síntomas asociados existen). El dolor de Marcelo aparece cuando camina o sube una escalera, se localiza en el pecho, se irradia a la mandíbula y ambos brazos, es opresivo, lo puntúa como de 7 a 8 sobre 10 , se atenúa rápidamente con el reposo y no cree identificar síntomas que se asocien. Me refiere que la noche anterior a la llamada, le dolió después de comer.

Marcelo era compañero mío de la escuela secundaria, hace 40 años, estudiábamos algunas materias juntos de noche en mi casa y ya fumaba 30 cigarrillos diarios.

Considerando que su dolor es anginoso típico y de acuerdo con los factores asociados, la probabilidad de que Marcelo tenga un síndrome coronario agudo supera ampliamente el 90 por ciento6. En ese contexto me encargué de averiguar qué cardiólogo había en Río Gallegos que hubiera hecho la residencia (no todos los médicos han hecho una residencia formal y no todos los especialistas se especializaron formalmente en la Argentina), lo llamé nuevamente a Marcelo y le dije que consultara al doctor Malatesta.

\section{Marcelo consultó a Malatesta.}

Malatesta lo vio en su consultorio y le solicitó una prueba ergométrica.

Marcelo tiene la obra social de la provincia a la que pertenece, por haber sido empleado público, pero tiene además una prepaga (un seguro privado) de cobertura nacional $y$, por habitar en suelo argentino, también puede ser atendido en cualquier centro del sistema público de salud.

A Marcelo se le dio un turno para realizar la prueba ergométrica en cinco días, pero al tercer día tuvo un infarto anterior. Fue derivado a Buenos Aires donde fue estudiado y revascularizado; actualmente recibe tratamiento médico e intenta dejar de fumar.

En el caso de Marcelo los "infortunios" que ocurrieron por llamarlos de una manera un tanto indulgente fueron: a) Ausencia de la complejidad necesaria o falta de acceso a la misma según los requerimientos del paciente; b) Impericia, imprudencia y negligencia médica (los tres componentes de la mala praxis).

A su vez, las causas o factores concurrentes de esta atención insuficiente y de mala calidad están determinados porque: a) La atención de la salud no está regionalizada y las fronteras en los diferentes niveles de atención son imprecisas; b) En la Argentina hay cientos de financiadores tanto del sector público, de las obras sociales sindicales o del sistema privado de salud. Estos sectores no están integrados, sus recursos no están planificados en forma integrada y muchas veces se superponen; c) La formación de los médicos es irregular y no está certificada.

La forma correcta de asistir a Marcelo era darle una aspirina inmediatamente, llamar a un sistema de emergencias (el doctor Malatesta, casualmente es uno de los dueños de un sistema de emergencias domiciliarias) y trasladarlo a un centro de alta complejidad. En ese centro debería haberse hecho una evaluación rápida, un electrocardiograma, evaluaciones enzimáticas y dada la inestabilidad, un cateterismo coronario con la posibi-lidad de colocar stents y, de ser necesario o surgir alguna complicación, realizar una cirugía de revascularización miocárdica a cielo abierto (bypass). El procedimiento era de urgencia y la prueba ergométrica estaba formalmente contraindicada.

\section{Niveles de atención y regionalización}

En el Reino Unido, cada habitante de su suelo está asignado a un médico generalista, es decir, toda la población tiene un médico de cabecera que constituye el primer y obligado nivel de atención y cada médico generalista tiene una determinada población a su cargo.

En la Argentina salvo raras excepciones, los pacientes no están asignados a un médico generalista ni los médicos generalistas tienen una población definida de pacientes. La proporción entre generalistas y especialistas no está regulada ni tampoco lo está la cantidad de especialistas o sub especialistas en función de la cantidad de población. Así, una ciudad de 100 mil habitantes puede tener escasos generalistas pero tres o cuatro neurocirujanos.

En la Argentina, la atención de la salud no está organizada por regiones; en algunas ciudades, como la que vive Marcelo suele decirse sarcásticamente que "el mejor médico es Aerolíneas Argentinas" (nuestra línea aérea de bandera).

En el Reino Unido la salud está organizada en diferentes niveles correspondientes a diferentes funciones específicas, roles, unidades administrativas y bases poblacionales. A determinada cantidad de población corresponde una determinada cantidad de médicos con una correspondiente cantidad y proporción de especialistas y una determinada cantidad y nivel de recursos hospitalarios.

Así, una población de 5 a 50 mil habitantes corresponde a un nivel de atención local y tiene cuidados de atención primaria; una localidad de entre 50 mil y 500 mil habitantes corresponde a un nivel de atención regional y tiene médicos y facilidades de atención primaria y secundaria y una población de entre 500 mil y 5 millones de habitantes tiene, además de los niveles primario y secundario, un tercer nivel de atención (Figura 1).

El nivel de atención primaria en el Reino Unido es dominio exclusivo de los médicos generalistas que se desempeñan en grupos de pequeño a mediano tamaño y cuya principal responsabilidad es los cuidados ambulatorios. Dos tercios de los médicos en el Reino Unido son médicos generalistas.

El segundo nivel de atención o nivel de cuidados secundarios está ocupado por especialistas en medicina interna, pediatras, neurólogos, psiquiatras, ginecólogos y obstetras y cirujanos generales. Estos médicos se desempeñan en hospitales y sirven como consultores de pacientes derivados del sector ambulatorio por los médicos generalistas. Los médicos del nivel secundario atienden también los pacientes internados. Una vez que terminan su atención los pacientes son retornados a sus médicos generalistas.

El nivel terciario de atención está ocupado por los sub especialistas como cirujanos cardiovasculares, inmunólogos, hematólogos pediátricos y atienden en unos pocos centros médicos de nivel terciario. 
De la misma forma la planificación de la atención hospitalaria sigue la misma lógica regional que la de los servicios médicos. Los hospitales distritales están equipados para servicios de internación básicos y los hospitales regionales manejan pacientes altamente especializados.

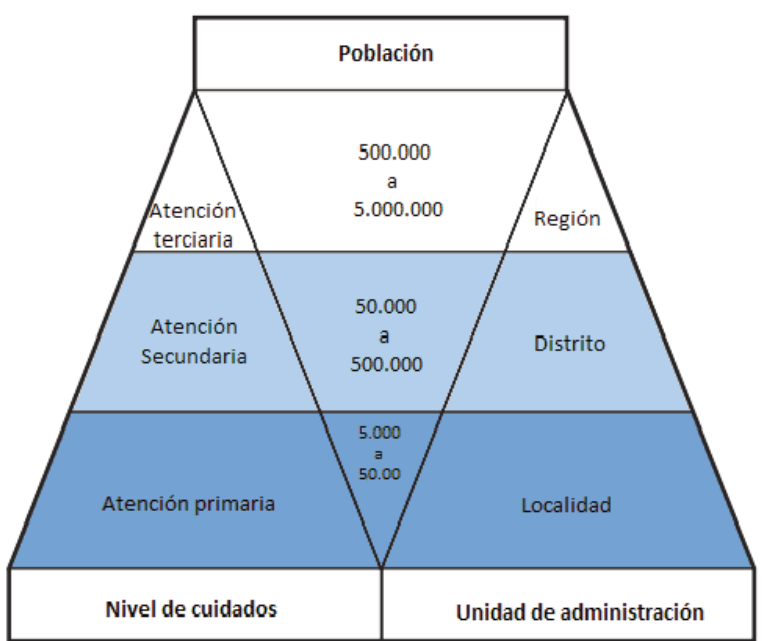

Figura 1. Organización en el tradicional modelo del Sistema Nacional de Salud del Reino Unido. La atención está estructurada en distintos niveles correspondientes a funciones específicas, roles, unidades administrativas y bases poblacionales. Tomado de Bodenheimer y Grumbach Understanding Health Policy: A clinical approach, 5th edition.

En la Argentina no existe la regionalización ni regulación del acceso a los diferentes niveles de atención. Dicho en otras palabras, un ciudadano que vive en Comodoro Rivadavia, Salta o Tartagal, puede trasladarse a la Ciudad Autónoma de Buenos Aires o Córdoba o Rosario y atenderse en cualquier hospital público y, si su financiador (su obra social sindical o provincial o entidad de medicina prepaga) se lo permite, concurrir a cualquier hospital público, hospital de comunidad o sanatorio privado.
Esto hace que los prestadores de salud (centros médicos, hospitales, clínicas y sanatorios) no puedan conocer la intensidad de la demanda y en consecuencia, regular los recursos para prestar servicios adecuadamente.

Así, una entidad prestadora de calidad y prestigio es muy demandada, es decir, no tiene una población definida a la que debe prestar sus servicios sino más bien, un universo infinito que puede concurrir a sus instalaciones y demandar sus servicios. En consecuencia sus camas serán siempre insuficientes, sus servicios de emergencia estarán continuamente desbordados, sus quirófanos trabajarán a pleno y sus pacientes en postoperatorio inmediato deberán aguardar horas hasta que en sus pisos o unidades de cuidados intensivos se libere una cama para ser internados.

\section{Conclusiones}

La falta de regionalización y atención por niveles en la Argentina, son dos de los varios componentes que hacen que la provisión de cuidados sea muy difícil de manejar en términos de satisfacción adecuada de la demanda, planificación, presupuestación y gestión de los servicios.

La población debería estar adecuadamente regionalizada y su accesibilidad asegurada, asimismo, la entrada a la prestación de servicios de salud que no son de emergencia debería hacerse a través del primer nivel de atención, el médico de familia, quien proveerá los cuidados necesarios y de ser pertinente establecerá la derivación a niveles superiores.

Cada individuo que habita el suelo argentino debería estar asignado a un médico de atención primaria y cada médico de atención primaria tener una población a cargo.

Recibido el 27/04/2016 y aceptado el 27/04/2016

\section{Referencias}

1. Centro Municipal de Cirugía Robótica de Malvinas [Sitio web]. Wikipedia. Disponible en URL: https://es.wikipedia.org/wiki/Centro_Municipal_de_Cirug\%C3\%ADa_Rob\%C3\%B3tica_de_Malvinas (último acceso abril 2016).

2. Ministerio de Salud de Misiones. La Provincia de Misiones ya cuenta con cirugía robótica Da Viunci en el Hospital escuela [Sitio web]. Consultor de Salud. Disponible en URL: http://consultordesalud.com.ar/detalle noticia.php?\&pais=Argentina\&tipo=\&idnoticia=125 (último acceso abril 2016).

3. Tan A, y col. Robotic surgery: disruptive innovation or unfulfilled promise? A systematic review and meta-analysis of the first 30 years. Surg Endosc. 2016 Feb 19 [Epub ahead of print]. Disponible en URL: http://www.ncbi.nlm.nih.gov/pubmed/26895896 (último acceso abril 2016).

4. The Commonwealth Fund. Mirror, mirror on the wall, 2014 update: how the US healthcare system compares internationaly [Sitio web]. 2014. Disponible en URL: http://www.commonwealthfund.org/publications/fund-reports/2014/jun/mirror-mirror (último acceso abril 2016).

5. Marshall M. A precious jewel--the role of general practice in the English NHS. N Engl J Med. 2015;372(10):893-7. Disponible en URL http://www.ncbi.nlm.nih.gov/pubmed/25738663 (último acceso abril 2016).

6. Simon D y Boring JR. Chapter 6 Sensitivity, Specificity, and Predictive Value. En: Clinical Methods: The History, Physical, and Laboratory Examinations. 3rd edition. 1990. Disponible en URL: http://www.ncbi.nlm.nih.gov/books/NBK383/ (último acceso abril 2016). 